\title{
RESILIENCIA DE LA COMUNIDAD EPÍGEA DE COLEOPTERA EN LAS LOMAS DE LACHAY DESPUÉS DEL EVENTO EL NIÑO 1997-98
}

\author{
Alfredo Giraldo Mendoza ${ }^{1}$ y Germán Arellano Cruz ${ }^{1}$
}

\begin{abstract}
Resumen
El presente estudio describe la resiliencia de una comunidad epígea de Coleoptera residente en formaciones de lomas ante los cambios provocados por la ocurrencia del evento El Niño 1997-98. La comunidad de Coleoptera fue evaluada 17 veces en el transcurso de cinco años $(1998$ - 2002) usando trampas pitfall que fueron sistemáticamente distribuidas en un área mixta de loma de herbáceas y loma tipo parque en la Reserva Nacional de Lachay. Para detectar las manifestaciones de la resiliencia a través del periodo de estudio fueron empleadas las siguientes medidas: abundancia, riqueza, diversidad, composición y abundancias relativas de las morfoespecies, abundancias relativas de los gremios, proporción carabidae:tenebrionidae $(\mathrm{C}: \mathrm{T})$ y un índice porcentual para medir estacionalidad (I.P.). Las manifestaciones más importantes de la resiliencia fueron detectadas al comparar las evaluaciones correspondientes al primer y segundo muestreo de cada año. Para las evaluaciones del 1er muestreo, fueron observadas tendencias progresivas de disminución de la diversidad, aumento de la abundancia relativa de saprófagos y aumento de la abundancia relativa de tenebriónidos. Para las evaluaciones del 2 do muestreo, se observaron diferencias marcadas entre la evaluación de 1998 y las evaluaciones de años posteriores, consistentes en una disminución en la diversidad, un aumento en la abundancia relativa de omnívoros y un aumento en la abundancia relativa de carábidos. La abundancia y la riqueza carecieron de patrones claros de resiliencia. Asimismo, la resiliencia significó un aumento progresivo de la estacionalidad, como fue indicado por los valores de I.P. calculados con la diversidad y la proporción $\mathrm{C}: \mathrm{T}$. La medida de resiliencia más efectiva fue la proporción $\mathrm{C}: \mathrm{T}$, por tal motivo se recomienda el uso de ésta y de otras proporciones de este tipo para la evaluación de perturbaciones.
\end{abstract}

Palabras clave: Comunidad epígea de coleópteros, resiliencia, El Niño 1997-98, formaciones de lomas, Reserva Nacional de Lachay.

\begin{abstract}
The present study describes the resilience of a epigeic beetle assemblage inhabiting in a lomas formations in the face of the changes caused by the occurrence of the El Niño 1997-98. The beetle assemblage was evaluated 17 times since 1998 until 2002 using pitfall traps that were systematically distributed in a mixed area of herbaceous lomas and type park lomas in the Lachay National Reserve. For detecting the manifestations of the resilience across the time, the following measures were employed: abundance, richness, diversity, composition and relative abundance of the morphospecies, relative abundance of the guilds, carabidae:tenebrionidae proportion $(\mathrm{C}: \mathrm{T})$ and a percentage index to measure seasonality (I.P.). The most important manifestations of the resilience were detected comparing the evaluations carried out in the 1 st and 2nd sampling of every year. For the 1st sampling evaluations, progressive tendencies of decrease of the diversity, increase of the relative abundance of detritivores and increase of the relative abundance of darkling beetles were observed. For the 2nd sampling, marked differences were observed between the 1998's evaluation and the evaluations of later years, these were a marked decrease in the diversity, an increase in the relative abundance of omnivores and an increase in the relative abundance of ground beetles. The abundance and the richness lacked clear patterns of resilience. Also, the resilience meant a progressive increase of the seasonality, as it was indicated by the I.P. values calculated with the diversity and the C:T proportion. The most effective measure of resilience was the $\mathrm{C}: \mathrm{T}$ proportion, for that reason the use of this kind of proportions is recommended for the evaluation of perturbations.
\end{abstract}

Key words: Beetle epigeic assemblage, resilience, El Niño 1997-98, lomas formations, Lachay National Reserve.

\section{Introducción}

Se denomina perturbación al desplazamiento de alguna propiedad de una comunidad o ecosistema lejos de su valor típico, el cual se considera generalmente que representa un valor de equilibrio o al menos un valor de estado estable para dicha

\footnotetext{
${ }^{1}$ Laboratorio de Control Biológico y Ecología de Artrópodos del Departamento Académico de Biología. Universidad Nacional Agraria La Molina. Av. La Molina s/n Apartado 456. Lima 100. Perú. Direcciones electrónicas: Giraldo troodon76@hotmail.com / Arellano acg@lamolina.edu.pe
} 
propiedad (Huston, 1994). El término disturbancia, que suele considerarse equivalente al de perturbación, es sólo un caso particular que consiste en cualquier proceso o condición externa a la fisiología natural de los organismos vivientes que produce una repentina pérdida de biomasa en una escala de tiempo significativamente más corta que la de su acumulación (Margalef, 1991; Huston, 1994). La respuesta de los sistemas afectados por tales cambios es la resiliencia, término que cuenta con un gran número de definiciones, las cuales pueden sintetizarse como "el retorno al estado o dinámica de referencia, después de una disturbancia temporal" (Grimm \& Wissel, 1997). Definiciones previas incluyen la planteada por Pimm (1984): "la rapidez con la cual las variables retornan hacia su equilibrio después de una perturbación" y aquella acuñada en el contexto del manejo adaptativo (Holling, 1996): "la habilidad de un sistema para absorber cambio y variación sin saltar a un estado diferente donde las variables y procesos que controlan su estructura y comportamiento cambien súbitamente."

Las lomas son formaciones vegetales que se desarrollan a modo de parches a lo largo del desierto hiperárido que se extiende en la costa oeste de Sudamérica $\left(5^{\circ} 00^{\prime}-29^{\circ} 55^{\prime}\right.$ L.S. $)$, debido a la confluencia de un complejo conjunto de condiciones físicas y meteorológicas que favorecen la condensación de neblinas en las elevaciones andinas cercanas al mar (Dillon \& Rundel, 1990). Tales condiciones persisten desde hace aproximadamente 5000 años, interrumpidas periódicamente por los eventos El Niño, los cuales producen un incremento significativo en las precipitaciones cada 2 o hasta 8.5 años (Rodbell et al., 1999). Cada uno de estos eventos constituye una oportunidad para la expansión de las lomas fuera de sus límites normalmente restringidos, lo cual hace de El Niño un elemento crucial en la dinámica y persistencia de estas formaciones vegetales (Dillon \& Rundel, 1990).

El área protegida de las lomas de Lachay ha sido el escenario de un numeroso conjunto de estudios acerca de los efectos de El Niño 1997-98 sobre las diferentes comunidades bióticas que habitan en dicho espacio (Alfaro et al., 2000). Más recientemente, la resiliencia de las comunidades de aves (Véliz, 2002) y de herbáceas (Tovar, 2003) han sido objeto de análisis detallado a partir de muestreos realizados por el Laboratorio de Ecología de Procesos - Dpto. de Biología (Universidad Nacional Agraria La Molina, Lima - Perú) entre los años 1998 y 2002. En tales muestreos, se colectó además abundante material de Coleoptera que sirvió para realizar una descripción de los patrones de variación espacio-temporal de dicha comunidad (Giraldo, 2002), en la cual el proceso de resiliencia sólo fue mencionado de modo implícito y en la que asimismo, no se incluyó las evaluaciones realizadas por el mismo equipo durante los meses de Junio y Octubre del 2002.

El presente trabajo se propone determinar las manifestaciones de la resiliencia en los patrones de variación temporal de la comunidad epígea de Coleoptera, frente a las perturbaciones provocadas por el evento El Niño 1997-98, a partir de datos obtenidos entre los años 1998 y 2002 en las lomas de Lachay.

\section{Materiales y métodos}

Área de estudio: Los muestreos fueron realizados en la Reserva Nacional de Lachay ubicada a $105 \mathrm{Kms}$. al norte de la ciudad de Lima $\left(11^{\circ} 21^{\prime} 00^{\prime \prime}-11^{\circ} 21^{\prime} 58^{\prime \prime}\right.$ L.S., $77^{\circ} 22^{\prime} 25^{\prime \prime}-77^{\circ} 22^{\prime} 28^{\prime \prime}$ L.O.). De su superficie total de 5,070 Ha, el área de estudio abarcó aproximadamente unas 430 ha comprendidas entre el Cerro Redondo, la Quebrada Yerbabuena y el Puquial, con un rango altitudinal de 200-600 m.s.n.m. Este espacio constituye una muestra representativa de las biocenosis denominadas loma de herbáceas y loma tipo parque (Brack, 1976).

Las condiciones meteorológicas típicas de la zona incluyen una temperatura invernal promedio de $13^{\circ} \mathrm{C}$, temperaturas veraniegas que superan $\operatorname{los} 25^{\circ} \mathrm{C}$ y precipitaciones que oscilan entre los 40 y $100 \mathrm{~mm}$ (Brack \& Mendiola, 2000). Los diferentes niveles de precipitación que se presentan a lo largo del año permiten distinguir cuatro épocas (Saito, 1976; Torres \& López Ocaña, 1981): seca (Enero - Marzo), inicios de época húmeda (Abril - Junio), época húmeda (Julio - Septiembre) e intermedia (Octubre - Diciembre).

Muestreo: Se realizaron cuatro evaluaciones por cada año para representar las épocas señaladas anteriormente, aunque durante los años 2001 y 2002 sólo pudieron realizarse tres y dos evaluaciones respectivamente. Se emplearon trampas pitfall para evaluar la comunidad de coleópteros presente en las 31 estaciones de muestreo que fueron distribuidas sistemáticamente dentro del área de estudio. Las trampas fueron envases de plástico transparente con un diámetro de $9 \mathrm{cms}$ y una capacidad aproximada de $572.5 \mathrm{~cm}^{3}$, enterrados al ras del suelo y llenos a la mitad de su capacidad con una mezcla de 7 partes de agua por 1 de formol al $40 \%$, además de un poco de detergente. El tamaño de la unidad muestral fue de dos trampas por estación con un esfuerzo temporal de siete días, el cual resultó satisfactorio para medir la diversidad de la entomofauna (Ramírez et al., 2002).

Los especimenes fueron contabilizados y registrados en su mayoría como morfoespecies, las cuales se asignaron a sus respectivas familias, empleando las claves de Britton (1973). No obstante, algunos de ellos pudieron determinarse a nivel de tribu, género o incluso especie, sea mediante comparación directa con ejemplares de colecciones ó mediante el uso de claves (Giraldo, 2002). Dichos especímenes se encuentran depositados en el Laboratorio de Control Biológico y Ecología de 
Artrópodos - Departamento de Biología de la Universidad Nacional Agraria La Molina Lima Perú (UNALM) y en el Museo de Entomología Klaus Raven de la misma institución.

Como registro de los efectos meteorológicos de El Niño se contó con los datos de precipitación total mensual, temperatura promedio mensual y humedad promedio mensual proporcionados por la estación meteorológica de la Reserva. Estos registros indican un aumento extraordinario de la precipitación durante 1998 en tanto, la temperatura y la humedad atmosférica no superaron el rango de años normales.

Análisis de los datos: Para evaluar el modo de acción de la resiliencia en la comunidad de Coleoptera se consideraron variables comunitarias, medidas de variación en las abundancias relativas de las morfoespecies y el índice porcentual propuesto por Tovar (2003).

Las variables comunitarias consideradas fueron la abundancia, la riqueza y la diversidad. La abundancia es el número de individuos capturados, la riqueza es el número de morfoespecies y la diversidad se calculó a partir de las abundancias de las morfoespecies usando el índice de Shannon (Krebs, 1989). Cada variable es presentada a través del promedio de los valores obtenidos en las 31 unidades muestrales por cada fecha de evaluación, los cuales cuentan con su respectivo intervalo de confianza calculado a partir de la distribución t (Steel \& Torrie, 1988).

Los cambios en las abundancias relativas se evaluaron a través de tres medidas diferentes: un análisis cluster, la variación en las abundancias relativas de gremios y la variación en la proporción carábidos: tenebriónidos.

El análisis cluster permitió agrupar las evaluaciones realizadas en base a la composición y abundancia relativa de las morfoespecies presentes en cada una de ellas. Los datos fueron transformados a la escala logarítmica, antes de ser procesados usando la técnica de unión promedio de clusters (average linkage clustering) y el coeficiente de correlación de Pearson (Krebs, 1989).

Las familias de coleópteros se agruparon en gremios, denominados categorías tróficas en Giraldo (2002), de acuerdo a las preferencias alimenticias predominantes de cada una de ellas (Britton, 1973; Crowson, 1981). Los cuatro gremios resultantes son: fitófagos, omnívoros, predatores y saprófagos. El gremio de fitófagos incluye consumidores de los diferentes órganos vegetales, el de predatores a especialistas y generalistas que actúan sobre diversos tipos de presas y, el de saprófagos a consumidores de cualquier tipo de materia orgánica en descomposición o de los microorganismos que habitan en ella. El gremio de omnívoros incluye sólo a los carábidos Notiobia peruviana Dejean y Pterostichus sp., puesto que incluyen materia vegetal en su dieta (Crowson, 1981; Noonan, 1981).

La proporción carábidos: tenebriónidos $(\mathrm{C}: \mathrm{T})$ se calculó a partir de las abundancias registradas para estas familias en cada fecha de evaluación. Una vez calculados, los valores fueron transformados a la escala logarítmica, de modo que valores positivos indicaran un predominio de carábidos, mientras que el caso contrario sea señalado por los valores negativos. Paoletti \& Cantarino (2000) han resaltado el uso de la proporción Carabidae: Tenebrionidae como un adecuado bioindicador de condiciones ambientales áridas, según el cual un predominio en la abundancia relativa de Tenebrionidae parece estar directamente relacionada a la prevalencia de condiciones áridas con pobre cobertura de vegetación.

El índice porcentual (I.P.) propuesto por Tovar (2003) es la diferencia porcentual existente entre los valores de una variable cualesquiera calculada para el 3er muestreo y el 1er muestreo de cada año. Este índice permite medir la estacionalidad comparando los dos periodos del año caracterizados por las condiciones meteorológicas más extremas en el curso de un año normal. Se expresa mediante la siguiente fórmula: I.P. $=$ (valor $3 \mathrm{er}$ muestreo- valor $1 \mathrm{er}$ muestreo) x 100/valor 3er muestreo. Las medidas consideradas en el cálculo de este índice son la abundancia, la riqueza, la diversidad y la proporción $\mathrm{C}: \mathrm{T}$ correspondientes a los cuatro primeros años, pues durante el año 2002 no se realizó la evaluación de la época seca.

El análisis cluster y las correlaciones que se requirieron fueron realizados con el programa Statistica versión 6.0 .

\section{Resultados y discusión}

La comunidad epigea de Coleoptera que fue muestreada incluyó 22,953 especímenes, correspondientes a 66 morfoespecies y 21 familias. Las familias dominantes, es decir las que incluyeron más del $85 \%$ de la abundancia total colectada fueron en orden descendente Tenebrionidae, Carabidae, Curculionidae, Staphylinidae y Elateridae. Las abundancias que presentaron las familias en cada evaluación se presentan en el Anexo 1.

Al comparar las abundancias promedio correspondientes a las evaluaciones realizadas en una misma época del año se observan las siguientes diferencias significativas $\mathrm{P} \leq 0.05$ (Figura 1): El mayor valor de Febrero-2001 con respecto a los demás del 1er muestreo, el mayor valor de Octubre-2002 con respecto a los demás del 3er muestreo, el menor valor de Agosto-1999 con respecto a los registrados en Septiembre-2001 y Octubre-2002 entre las del 3er muestreo, así como el menor valor de Diciembre-1999 con respecto a los demás del 4to muestreo. En ninguno de los casos se observan tendencias 
RESILIENCIA DE COLEOPTERA EN LACHAY DESPUÉS DEL EVENTO EL NIÑO 1997-98 Diciembre 2003

Figura $\mathrm{n}^{\circ}$ 1. Cambios en la abundancia promedio de la comunidad de Coleoptera de cada muestreo entre los años 1998 y 2002. Intervalos de confianza a partir de la distribución t $(\alpha=0.05)$.

(a) 1er muestreo

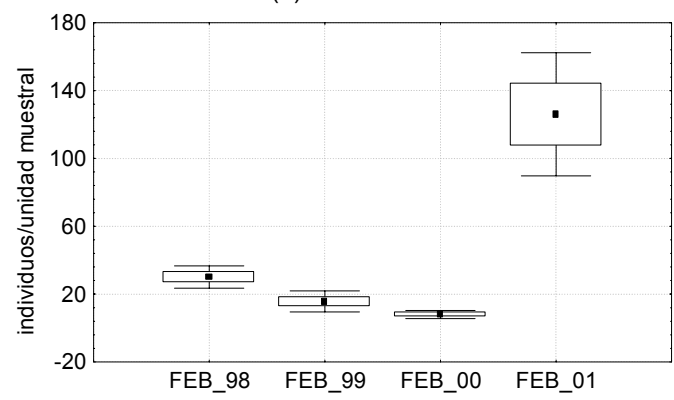

(c) 3er muestreo

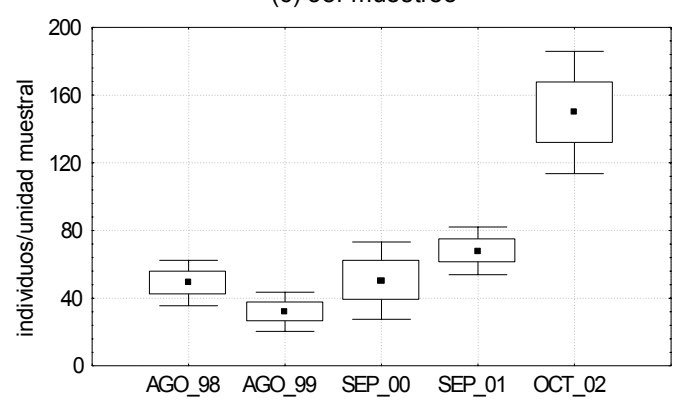

(b) 2do muestreo

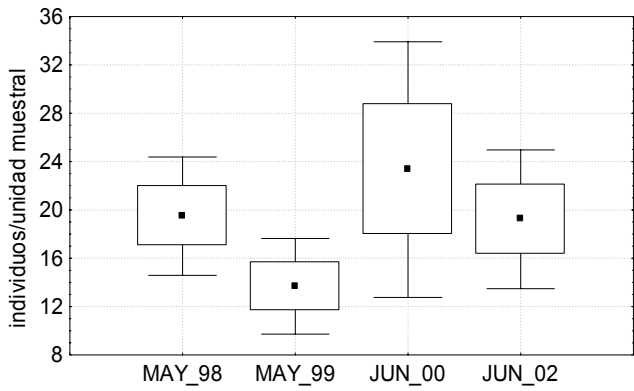

(d) 4to muestreo

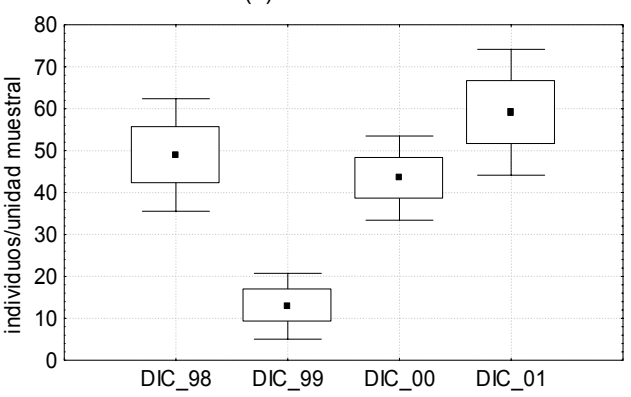

Figura $\mathbf{n}^{\circ}$ 2. Cambios en la riqueza promedio de la comunidad de Coleoptera de cada muestreo entre los años 1998 y 2002. Intervalos de confianza a partir de la distribución t ( $\alpha=0.05)$.

(a) 1er muestreo

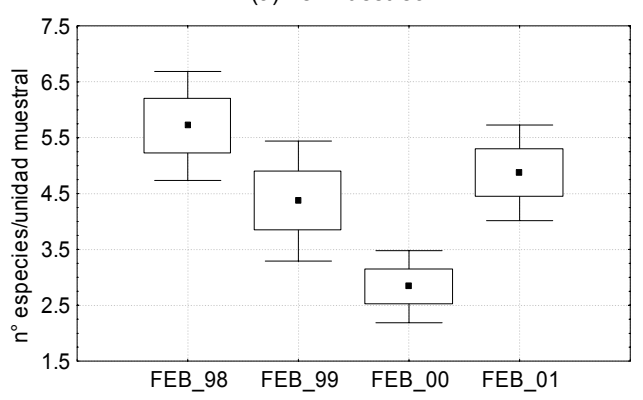

(c) 3er muestreo

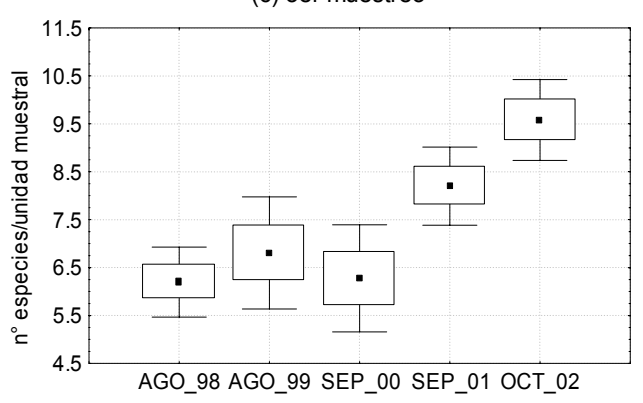

(b) 2do muestreo

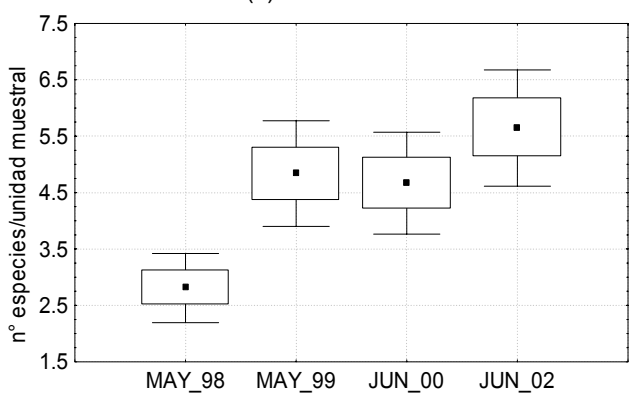

(d) 4 to muestreo

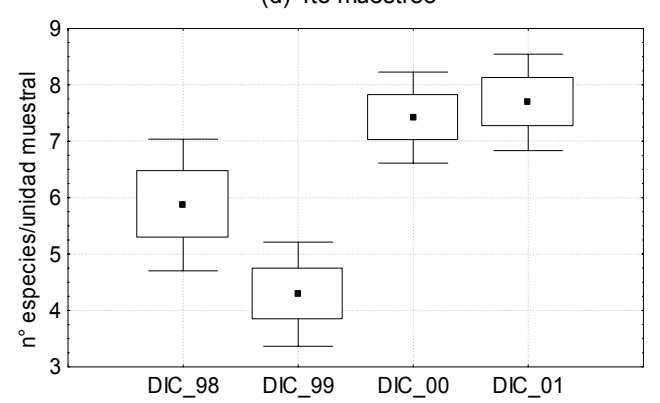


Figura $\mathbf{n}^{\circ}$ 3. Cambios en la diversidad promedio de la comunidad de Coleoptera de cada muestreo entre los años 1998 y 2002. Intervalos de confianza a partir de la distribución t $(\alpha=0.05)$.

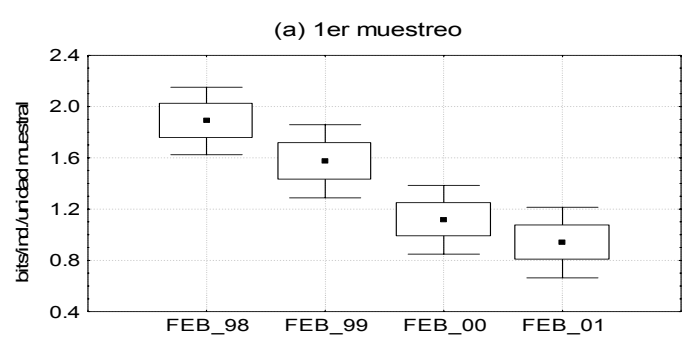

(c) 3er muestreo

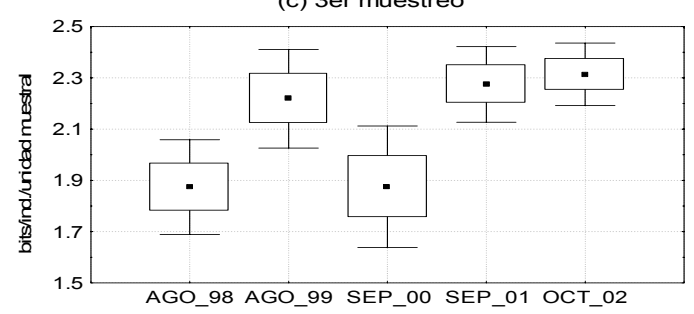

ascendentes o descendentes que cubran todo el periodo de evaluación.

Al comparar las riquezas promedio correspondientes a las evaluaciones realizadas en una misma época del año se observan las siguientes diferencias significativas $\mathrm{P} \leq 0.05$ (Figura 2): el menor valor de Febrero-2000 con respecto al de Febrero2001 entre los del 1er muestreo, el menor valor de Mayo-1998 con respecto a los demás del 2do muestreo, el menor valor de Agosto-1998 con respecto a los registrados en Septiembre-2001 y Octubre-2002 entre los del 3er muestreo, además de el menor valor de Diciembre-1999 con respecto a los registrados en Diciembre-2000 y Diciembre-2001 entre los del 4to muestreo. En ninguno de los casos se observan tendencias ascendentes o descendentes que cubran todo el periodo de evaluación.

Al comparar las diversidades promedio correspondientes a las evaluaciones realizadas en una misma época del año se observan las siguientes diferencias significativas $\mathrm{P} \leq 0.05$ (Figura 3): El mayor valor de Febrero1998 con respecto a los registrados en Febrero-2000 y Febrero-2001 entre los del 1er muestreo, el menor valor de Mayo-1998 con respecto a los demás del 2do muestreo y asimismo, los menores valores de Agosto-1998 y Septiembre-2000 con respecto a los registrados en Septiembre-2001 y Octubre-2002. En el caso del primer muestreo se observa una tendencia descendente que cubre todo el periodo de evaluación.

En el dendrograma elaborado con los datos de abundancia de las

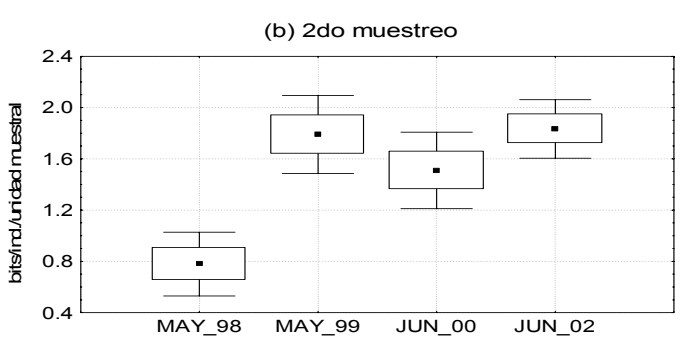

(d) 4 to muestreo

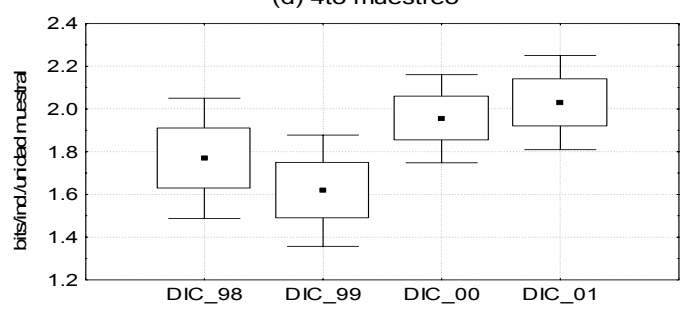

morfoespecies (Figura 4), a una distancia de unión de 0.4 se distinguen cinco asociaciones: La $1 \mathrm{ra}$ asociación incluye a todas las evaluaciones realizadas durante 1998, la 2da asociación incluye las evaluaciones de Mayo,

Agosto y Diciembre de 1999, la 3ra asociación incluye las evaluaciones correspondientes al 3er y 4to muestreo de los últimos tres años, la 4ta asociación incluye las evaluaciones correspondientes al 2do muestreo de los años 2000 y 2002. y finalmente, la 5ta asociación incluye las evaluaciones correspondientes al 1er muestreo de 1999, 2000 y 2001. Al interior de la 3ra asociación se distinguen claramente dos grupos, uno correspondiente al 3er muestreo y el otro al 4to muestreo. Asimismo, en la 5ta asociación existe una distinción muy marcada entre la evaluación de

Figura $n^{\circ} 4$. Dendrograma de las 17 fechas de evaluación (matriz de abundancia de morfoespecies) Coeficiente de correlación de Pearson

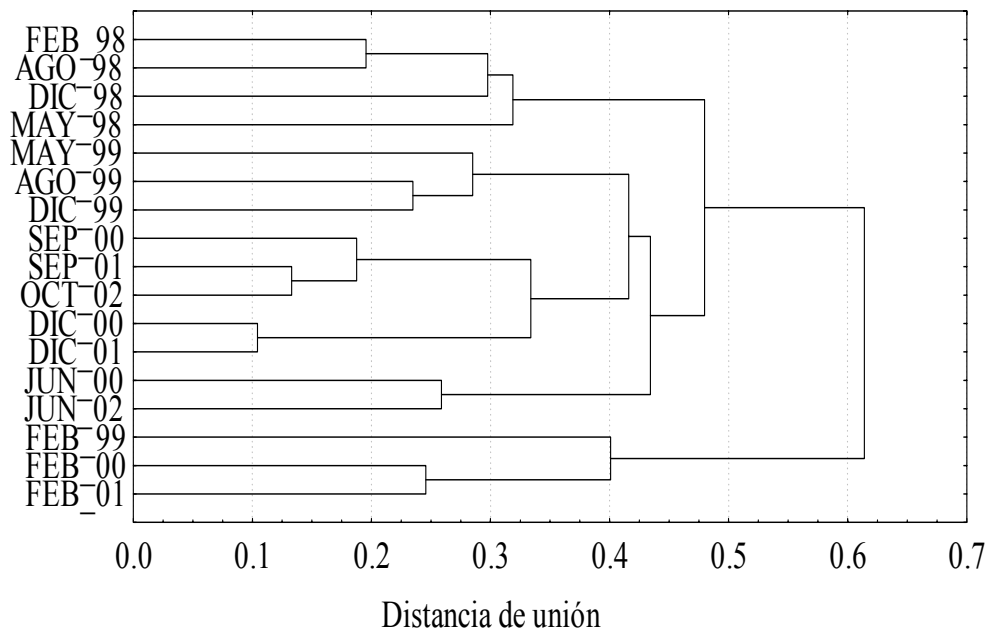


Febrero-1999 y las otros dos. Resulta importante señalar que las distancias de unión al interior de la 3ra, 4 ta y 5 ta asociación, presentan diferentes magnitudes.

Dichas magnitudes son mayores entre las evaluaciones correspondientes al 1er y 2 do muestreo, que entre las evaluaciones del 3er muestreo y más aún con respecto a la existente entre las evaluaciones del 4 to muestreo.

Figura $n^{\circ}$ 5. Cambios en la abundancias relativas de los gremios de la comunidad de Coleoptera de cada muestreo entre los años 1998 y 2002. Ft=fitófagos, Om= omnívoros, $\mathrm{Pr}=$ predatores, $\mathrm{Sp}=$ saprófagos.
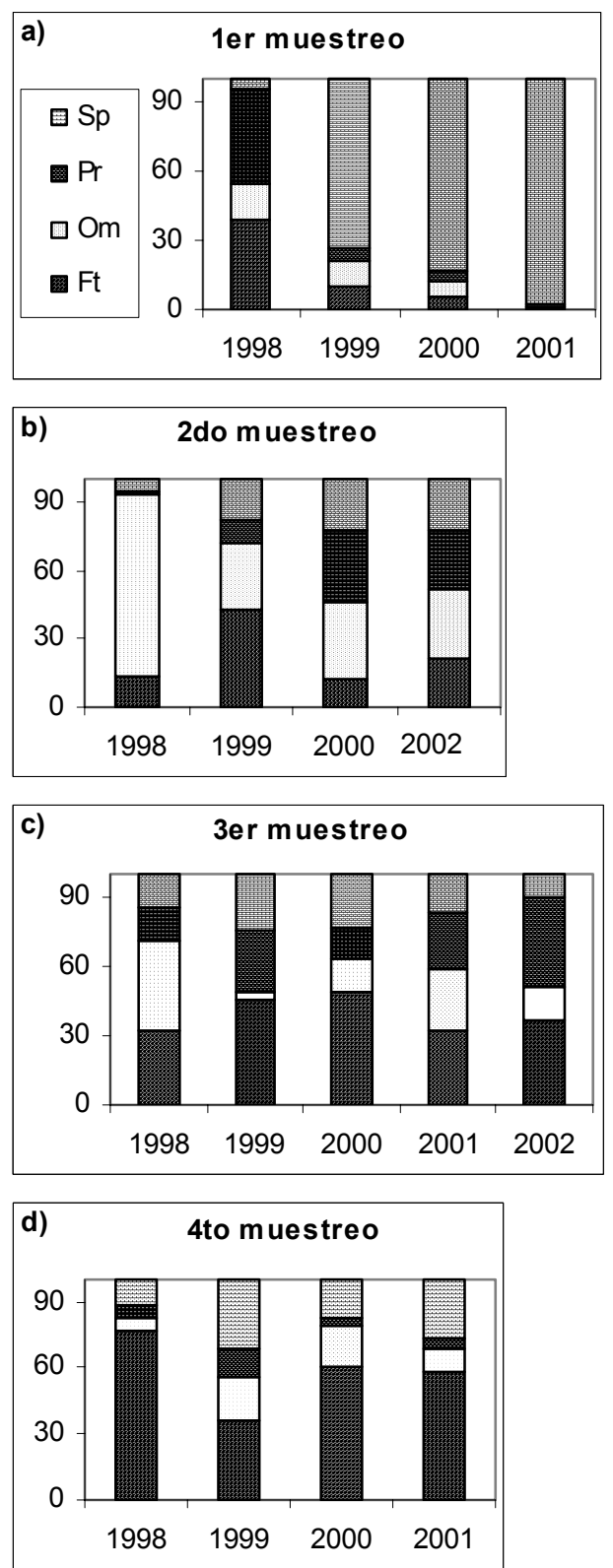

Al comparar las abundancias relativas de los gremios (Figura 5) se observan los siguientes cambios entre las evaluaciones correspondientes a cada muestreo: Entre las evaluaciones del 1er muestreo, la de 1998 se distinguió por el predominio compartido de fitófagos y predatores, en contraste con el predominio de saprófagos registrado durante los años 1999, 2000 y 2001. Entre las evaluaciones del 2do muestreo, la de 1998 presentó un predominio de omnívoros, la de 1999 un predominio compartido de fitófagos y predatores, mientras que las de los años 2000 y 2001 se caracterizaron por un predominio compartido de omnívoros y predatores. Entre las evaluaciones del 3er muestreo, las de 1998 y 2001 presentaron un predominio compartido de fitófagos y omnívoros, las de 1999 y 2002 un predominio compartido de fitófagos y predatores, mientras que la del año 2000 un predominio compartido de fitófagos y saprófagos. Entre las evaluaciones del 4to muestreo, la de 1998 presentó un predominio de fitófagos, las de 1999 y 2001 un predominio compartido de fitófagos y saprófagos, en tanto la del año 2000 presentó un predominio compartido de fitófagos y predatores.

Al comparar las proporciones carábidos: tenebriónidos (Figura 6) se observan los siguientes cambios entre las evaluaciones correspondientes a cada muestreo: Entre las evaluaciones del 1er muestreo, la de 1998 se distinguió por un alto valor positivo, en contraste con los valores cada vez más negativos registrados para los años 1999, 2000 y 2001. Todas las evaluaciones del 2 do muestreo presentaron valores positivos con una clara tendencia descendente desde 1998 hasta el 2002. Entre las evaluaciones del 3er muestreo, la de 1998 se distinguió por su altor valor positivo, en tanto el resto de evaluaciones mostraron valores positivos ó negativos muy cercanos o incluso iguales a cero (año 2000). Todas las evaluaciones del 4to muestreo presentaron valores positivos o negativos cercanos a cero.

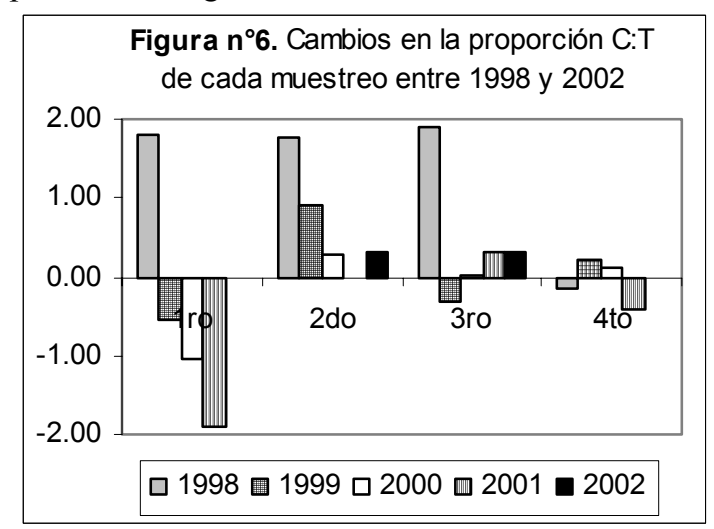

El índice porcentual (Figura 7) muestra una tendencia ascendente y continua durante el periodo 1998 - 2001, cuando es calculado a partir de los valores de diversidad promedio y de la proporción $\mathrm{C}: \mathrm{T}$. En cambio, cuando éste es calculado a partir de los valores de riqueza promedio, dicha tendencia se interrumpe en el año 2000 por el menor valor del índice obtenido para el año 2001. En el caso de la 
abundancia promedio, se observa la misma tendencia pero fuertemente alterada por el valor negativo que adquirió el índice el año 2001. En todos los casos, obviando el signo del índice, la diferencia entre los valores de las distintas medidas empleadas aumentó a través del tiempo.

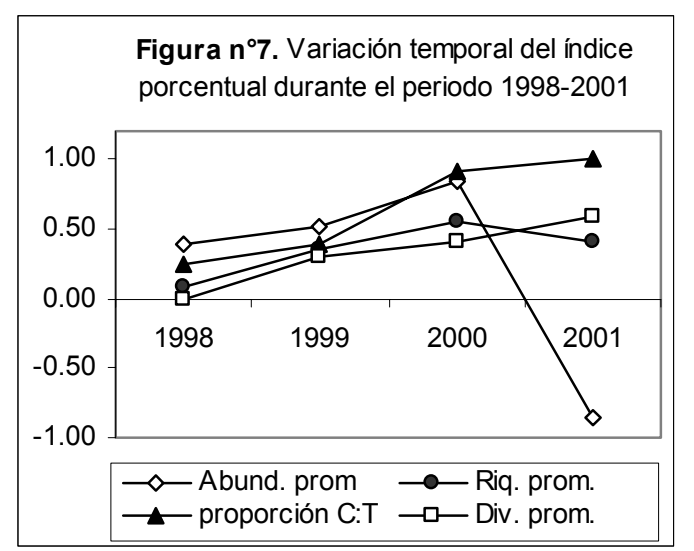

En función de los resultados expuestos anteriormente, las manifestaciones de la resiliencia en la comunidad examinada pueden sintetizarse en:

- Para la diversidad se distinguieron tres manifestaciones distintas, dependientes del porcentaje de abundancia alcanzado por las especies dominantes, las cuales incluyen no menos del $30 \%$ de la abundancia por muestreo. Entre las evaluaciones del ler muestreo, se produjo una pérdida gradual de equidad acompañada por una serie de cambios en las especies dominantes: dominancia del carábido Calosoma rufipenne Dejean (Febrero-1998), dominancia compartida del tenebriónido Ammophorus rubripes Solier y el clámbido Clambus sp. (Febrero1999) y dominancia del tenebriónido Evaniosomus orbygnianus Guerin (Febrero-2000 y Febrero-2001). En el caso de las evaluaciones del 2do muestreo, las condiciones cambiaron desde una con baja equidad provocada por la dominancia del carábido Pterostichus sp. (Mayo-1998) hasta alcanzar niveles de mayor equidad, en los cuales se presentaron dominancias compartidas de Pterostichus $s p$. con una especie indeterminada de Elateridae (1999) y una de Coccinellidae (2000 y 2002). Para las evaluaciones del 3er muestreo, se produjeron dos descensos debido a las dominancias de Pterostichus sp. (1998) y de una especie indeterminada de Curculionidae - Naupactini (2000) respectivamente.

- Para la composición y abundancias relativas de las morfoespecies se observó un retorno a las condiciones que corresponden normalmente a cada estación del año, tal como queda ilustrado por la 3ra (época húmeda e intermedia), 4ta (inicios de época húmeda) y 5ta (época seca) asociaciones. Todo ello luego de atravesar por dos etapas anómalas, la 1ra asociación (todo el año 1998) y la 2da asociación (el año 1999 excepto por Febrero). Dichas asociaciones responden en última instancia a los ciclos vitales y niveles de actividad anual que caracterizan a las poblaciones durante un año con estacionalidad normal, los cuales deben reorganizarse en función de los cambios provocados por el EN.

- Para las abundancias relativas de los gremios, se observó también un retorno a las condiciones que corresponden normalmente a cada estación del año. Para las evaluaciones correspondientes al 2do, 3er y 4to muestreo significó un descenso en el predominio de gremios cuya abundancia relativa es importante en años normales, pero claramente menor a la alcanzada durante 1998 ó 1999. Los casos destacables al respecto, están dados por el predominio de omnívoros (Mayo-1998) y el de fitófagos (Diciembre-1998). Para las evaluaciones correspondientes al 1er muestreo significó la recuperación del predominio del gremio de saprófagos, tras haber sido desplazados por los tres gremios restantes durante 1998. En consecuencia, las condiciones ambientales con un balance hídrico favorable y consiguiente incremento en las estructuras vegetales (por incidencia estacional de neblinas $o$ precipitaciones provocadas por El Niño) son requisitos para la proliferación de los gremios de fitófagos, omnívoros y predatores. En cambio, el gremio de saprófagos encuentra condiciones para su proliferación en la época seca de años normales, cuando queda disponible gran cantidad de materia vegetal en estado de descomposición.

- Para la proporción C:T, la manifestación de la resiliencia fue bastante clara, el retorno de una condición en la cual los tenebriónidos predominan sobre los carábidos durante la época seca y con las abundancias de ambas familias casi equiparadas durante el resto del año, en lugar del notorio predominio de carábidos observado en las tres primeras evaluaciones de 1998. La clara manifestación de la resiliencia a través de esta proporción, no es sorprendente puesto que los cambios en la diversidad señalados anteriormente tuvieron como causa principal cambios en las abundancias de algunas especies de carábidos y tenebriónidos. Asimismo, con respecto a las abundancias registradas para los gremios, los carábidos tuvieron una contribución importante en el gremio de predatores y fueron integrantes exclusivos del gremio de omnívoros, en tanto los tenebriónidos constituyeron el componente mayoritario del gremio de saprófagos.

- Para el índice porcentual (I.P.), la resiliencia se manifestó en un aumento progresivo de valores, el cual señala que la estacionalidad fue alterada y que la recuperación de ésta, al menos para la diversidad y la proporción $\mathrm{C}: \mathrm{T}$, no había concluido transcurridos tres años desde la ocurrencia de El Niño.

Para la abundancia y la riqueza, resulta difícil distinguir un patrón de resiliencia claro debido a la frecuencia con la cual los intervalos de confianza de sus valores se traslapan y, a la ausencia de tendencias 
que cubran todo el periodo de estudio. No obstante, es posible sugerir dos patrones para estas variables comunitarias: una tendencia al incremento de valores hacia el final del periodo de evaluación y, la ocurrencia de los menores valores para el año 1999 e inicios del 2000. El primer patrón se ve ilustrado por el 1er y 3 er muestreo para la abundancia y, por el 2 do, 3er y 4to muestreo para la riqueza. El segundo patrón es válido para la abundancia entre Mayo-1999 y Febrero-2000, mientras que para la riqueza es válido sólo para Diciembre-1999 y Febrero-2000.

De los patrones propuestos, el primero de ellos resulta contradictorio para la abundancia puesto que se esperaba una respuesta positiva acorde con el aumento de cobertura vegetal propiciado por El Niño (Tovar, 2003). La relación directa entre abundancia de artrópodos y diferentes medidas de desarrollo vegetal ha sido observada previamente, así por ejemplo con la estructura vertical de la vegetación (Dennis et al., 1998) ó con la productividad primaria (Ayal \& Merkl, 1994). Para la riqueza, el primer patrón implicaría que la mayor variabilidad meteorológica de un año normal estaría ofreciendo un escenario adecuado para la coexistencia de más especies que un lapso de tiempo meteorológicamente homogéneo provocado por El Niño. El segundo patrón parece indicar que estas variables fueron negativamente afectadas por los bajos niveles de precipitación que caracterizaron a 1999, aunque como se indica más adelante sus correlaciones con la precipitación carecen de significación estadística.

Pueden distinguirse tres características importantes en la resiliencia de la comunidad de coleópteros. Primero, los efectos son discernibles sobretodo en los muestreos correspondientes a la primera mitad del año. Esto guarda correspondencia con la duración de las precipitaciones provocadas por El Niño, las cuales sobrepasaron los niveles normales hasta Agosto de 1998. Segundo, la duración del proceso de resiliencia puede fijarse en dos años, es decir el año 2000 puede considerarse como un año normal, aunque no obstante las diferentes medidas empleadas indican que la estacionalidad se acentuó durante los años 2001 y 2002. Tercero, las variables comunitarias se mostraron menos sensibles a la perturbación de El Niño que las medidas de cambio en las abundancias relativas. Así lo demuestran las tendencias obtenidas con el índice porcentual (I.P.), así como la correlación de éstas con la precipitación total mensual, la cual es significativa para la proporción $\mathrm{C}: \mathrm{T}(\mathrm{r}=0.69, \mathrm{p}=0.005)$ y no significativa para las variables comunitarias (abundancia $\mathrm{r}=-0.04, \mathrm{p}=0.89$; riqueza $\mathrm{r}=0.28, \mathrm{p}=$ 0.32 ; diversidad $\mathrm{r}=0.35, \mathrm{p}=0.19$ ).

Es preciso indicar, que si bien la precipitación es indicada frecuentemente como el agente causal de los patrones observados, el establecimiento formal de una relación causa-efecto exigiría en este caso establecer la demora de respuesta (time-lag) de la comunidad de
Coleoptera frente a esta variable meteorológica. Para tal efecto, se hubiera requerido un muestreo continuo cuyos datos servirían para efectuar un análisis de series de tiempo. Longcore (1999) reporta que la abundancia media mensual de 50 morfoespecies de artrópodos epigeos, tuvo en promedio una demora de respuesta de $2.8 \pm 2.1$ meses, con respecto a la precipitación total mensual en un ambiente costero del sur de California.

La diferente sensibilidad de variables comunitarias y medidas de cambio en las abundancias relativas frente a la ocurrencia de perturbaciones, ha sido previamente reconocida en otras comunidades de coleópteros epígeos. Es el caso de Werner \& Raffa (2000) y Jankielsohn et al. (2001), quienes afirman que la composición y/o los cambios en las abundancias relativas fueron más sensitivas para caracterizar los tipos de hábitat y los regímenes de manejo evaluados por ellos, que la riqueza específica, la equidad o la diversidad medida con los índices de Simpson y Shannon.

Comparando de modo preliminar la resiliencia de la comunidad epigea de Coleoptera con las descritas para las otras comunidades evaluadas simultáneamente en las lomas de Lachay, se observa como principal diferencia que las variables comunitarias fueron más sensibles, así en el caso de la vegetación herbácea (Tovar, 2003) la densidad, la cobertura y la diversidad estuvieron correlacionadas significativamente con la precipitación y, en el caso de la avifauna (Véliz, 2002) la abundancia, la riqueza y la diversidad aumentaron de modo notable durante 1998. A semejanza de la comunidad de Coleoptera, en la comunidad de herbáceas se produjeron incrementos en la abundancia relativa de algunas especies, en este caso las solanáceas Nicotiana paniculata, Nolana humifusa y Lycopersicon peruvianum (Cano et al., 1999). En tanto, la comunidad de aves fue similar a la de Coleoptera en vista de los incrementos registrados en las abundancias relativas de algunos gremios durante 1998, en este caso fueron los gremios de insectívoros y predatores (Véliz, 2002). Por otra parte, para la comunidad de arañas epigeas se cuenta sólo con un primer avance acerca de su resiliencia, en base al análisis de los datos correspondientes a 1998 y 1999 (en este volumen).

La proporción Carabidae: Tenebrionidae se destaca como una eficiente medida del grado de aridez ambiental, no sólo porque en el presente caso tuvo resultados más claros que las variables comunitarias, sino además porque se fundamenta en las distinta relación que cada una de estas familias ha establecido con respecto a los recursos hídricos disponibles en los ambientes terrestres. En efecto, los carábidos han tenido su mayor radiación adaptativa en ecosistemas húmedos, siendo su diversidad menor en ambientes templados áridos (Erwin, 1985). Esto guarda relación con el linaje ancestral de carábidos, cuyo hábitat 
habría sido las áreas ribereñas de ecosistemas tropicales húmedos durante el Pérmico (Erwin, 1979).

Por su parte, los tenebriónidos han llegado a ser el grupo dominante de coleópteros en todas las regiones desérticas del mundo (Britton, 1973; Crowson, 1981), paralelamente al desarrollo de un conjunto de adaptaciones de tipo estructural, etológico, fisiológico y morfométrico, cuyas expresiones más notables se encuentran entre las especies del desierto de Namib (Hamilton \& Seely, 1976; Crowson, 1981; Nicolson et al., 1984; Nicolson, 1990).

Finalmente, cabe señalar que proporciones tales como la $\mathrm{C}: \mathrm{T}$ presentan ventajas metodológicas en relación a las otras medidas de variación en las abundancias relativas. Dichas ventajas son la capacidad de ser expresadas mediante una sola cifra (fácil de graficar y susceptible de contar con un intervalo de confianza) y además, la reducción considerable del esfuerzo que demanda la determinación de numerosas familias (con sus respectivas especies) ó la formación de gremios (que implica muchas veces atribuir hábitos generales a especies cuya historia natural es desconocida). Por ello, para la evaluación de perturbaciones resultaría conveniente el uso de alguna proporción que confronte la abundancia de grupos taxonómicos o funcionales de requerimientos opuestos frente a una variable ambiental impactada.

\section{Conclusiones}

La resiliencia de la comunidad epígea de Coleoptera frente a El Niño 1997-98 estuvo definida por la recuperación de los patrones de variación temporal típicos de las diferentes especies a lo largo del año, es decir la estacionalidad que caracteriza normalmente sus ciclos vitales y niveles de actividad anual. Dichos patrones fueron alterados notoriamente entre Febrero y Agosto de 1998, produciéndose con respecto a las condiciones de un año normal, cambios ascendentes y descendentes en los valores de diversidad, desplazamiento del gremio de saprófagos en favor del resto de gremios y aumentos en la proporción C:T. Sin embargo, los cambios abarcaron los dos primeros años cuando se examinan a través de las abundancias relativas de las morfoespecies y los gremios. Mas aún, al menos para la diversidad y la proporción $\mathrm{C}: \mathrm{T}$, la recuperación de la estacionalidad parece ser un proceso inconcluso luego de transcurridos tres años a partir de la ocurrencia de dicho evento.

\section{Agradecimientos}

Al Consejo Nacional de Ciencia y Tecnología (CONCYTEC) por el financiamiento otorgado al proyecto en el marco de la Red de Impacto Biológico "El Niño" RIBEN. Al personal de la Reserva Nacional de Lachay, por las facilidades brindadas para la realización de las evaluaciones durante las gestiones de David Velarde e Iris Zárate.

\section{Literatura citada}

Alfaro L., Barrera R. \& Velarde D. 2000. Reporte Lachay. INRENA-Dirección General de Áreas Naturales Protegidas y Fauna Silvestre. Lima Perú.

Ayal Y. \& Merkl O. 1994. Spatial and temporal distribution of tenebrionid species (Coleoptera) in the Negev Highlands, Israel. Journal of Arid Environments. 27: 347-361.

Brack A.J. 1976. Ecología Animal. Primera Parte: Sinecología. Editor Pedro Aguilar F.

. \& Mendiola C. 2000. Ecología del Perú. Programa de las naciones unidas para el desarrollo (PNUD). Editorial Bruño. Lima-Perú.

Britton E.B. 1973. Coleoptera (Beetles). In: The Insects of Australia. Melbourne University Press.

Cano A., Roque J., Arakaki M., Arana C., La Torre M., Llerena N. \& Refulio N. 1999. Diversidad florística de las Lomas de Lachay (Lima) durante el evento "El Niño 1997-98". Revista Peruana de Biología, Vol.Extraordinario.: 125-132.

Crowson R.A. 1981. The Biology of the Coleoptera. Academic Press Inc. (London).

Dennis P., Young M.R \& Gordon I.J. 1998. Distribution and abundance of small insects and arachnids in relation to structural heterogeneity of grazed, indigenous grasslands. Ecological Entomology. 23(3): 253-264.

Dillon M.O. \& Rundel P.W. 1990. The botanical response of the Atacama and peruvian desert floras to the 1982-83 El Niño Event. En: Global Ecological Consequences of the 1982-83 El NiñoSouthern Oscillation. Glynn, P.W. (ed.) Elsevier, New York.

Erwin T.L. 1979. Thoughts on the evolutionary history of ground beetles: hypoteses generated from comparative faunal analyses of lowland forest sites in temperate and tropical regions. En: Erwin TL, GE Ball \& DR Whitehead (eds) Carabid beetles, their evolution, natural history, and classification: 539-592. Dr. W. Junk Publishers, The Hague, The Netherlands. 1985. The taxon pulse: a general pattern of lineage radiation and extinction among carabid beetles. En: Ball GE (ed) Taxonomy, phylogeny and biogeography of beetles and ants: 437-488. Dr. W. Junk Publisher, The Hague, The Netherlands.

Giraldo A. 2002. Análisis de los patrones de variación espacio-temporal de las poblaciones de coleópteros en la Reserva Nacional de Lachay durante el periodo 1998 - 2001. Tesis para optar el Título de Biólogo - UNALM.

Grimm V. \& Wissel C. 1997. Babel, or the ecological stability discussions: an inventory and analysis of 
terminology and a guide for avoiding confusion. Oecologia. 109: 323-334.

Hamilton W.J. \& Seely M. 1976. Fog basking by the Namib Desert beetle, Onymacris unguicularis. Nature. 262: 284-285.

Holling C.S. 1996. Surprise for science, resilience for ecosystems, and incentives for people. Ecological Applications. 6(3): 733-735.

Huston H. 1994. Biological diversity, the coexistance of species in changing landscapes. Cambridge University Press.

Jankielshon A., Scholtz C.H. \& Louw S. 2001. Effect of habitat transformation on Dung beetle assemblages: A comparison between a South African Nature Reserve and neighboring farms. Environmental Entomology. 30(3): 474-483.

Krebs J. 1989. Ecological Methodology. University of British Columbia. Harper Collins Publishers.

Longcore T.R. 1999. Terrestrial Arthropods as Indicators of Restoration Success in Coastal Sage Scrub. A dissertation for the degree Doctor of Philosophy in Geography. University of California- Los Angeles.

Margalef R. 1991. Reflexiones sobre la Diversidad y significado de su expresión cuantitativa. Diversidad Biológica: 105-114. Fundación Araces, Madrid.

Nicolson S. 1990. Water relationships of the Namib tenebrionid beetles. En Seely, M. K. ed., Namib ecology: 25 years of Namib research. Transvaal Museum Monograph. 7: 173-178.

, Bartholomew G.A. \& Seely M.K. 1984 Ecological correlates of locomotion speed, morphometrics and body temperature in three Namib Desert tenebrionid beetles. S. Afr. Tydskr. Dierk. 19(3): 131-134.

Noonan G. 1981. South American species of the subgenus Anisotarsus Chaudoir (genus Notiobia Perty: Carabidae: Coleoptera). Part I: Taxonomy and Natural History. Contributions in Biology and Geology, 44: 1-84. Milwaukee Public Museum.

Paoletti M.G. \& Cantarino C.M. 2000. The use of invertebrates in evaluating rural sustainability.
Chapter 3 in: Interchanges of Insects. M. Irwin and Y. Robert (eds.). : 33-52.

Pimm S.L. 1984. The complexity and stability of ecosystems. Nature. 307: $321-326$.

Ramírez D., Pérez D., Sánchez E. \& Arellano G. 2002. Esfuerzo de muestreo para la evaluación de la diversidad colectada en pitfall en la Reserva Nacional de Lachay-Perú. Ecología Aplicada. 1(1): 37-42.

Rodbell D.T., Seltzer G.O., Anderson D. M., Abott M. B., Enfield D.B. \& Newman J.H. 1999. An 15000 year record of El Niño - driven alluviation in southwestern Ecuador. Science. 283: 516 - 520.

Saito C. 1976. Bases para el establecimiento y manejo de una unidad de conservación en las Lomas de Lachay, Perú. Tesis para optar el título de Ingeniero Forestal- Universidad Nacional Agraria La Molina, Lima Perú.

Steel R. \& Torrie J. 1988. Bioestadística: Principios y procedimientos. Mc Graw-Hill Interamericana de México S.A. de C.V.

Torres J. \& López Ocaña C. 1981. Productividad primaria en las Lomas de la Costa Central del Perú. Separata del Boletín de Lima. (14): 1 - 11.

Tovar C. 2003. Análisis de la Resiliencia de las comunidades herbáceas de las Lomas de Lachay, provincia de Huaura, Departamento de Lima después de su perturbación por el evento El Niño 1997-1998. Tesis para optar el Título de Bióloga UNALM.

Véliz C. 2002. Resiliencia de comunidades de aves en la Reserva Nacional de Lachay luego del evento El Niño 1997 - 98. Tesis para optar el Título de Bióloga , Universidad Nacional Agraria La Molina, Lima - Perú.

Werner S.M. \& Raffa K.F. 2000. Effects of forest management practices on the diversity of groundocurring beetles in mixed northern hardwood forest of the Great Lakes Region. Forest Ecology and Management. 139: 135-155.

\begin{tabular}{|c|c|c|c|c|c|c|c|c|c|c|c|c|c|c|c|c|c|c|}
\hline \multirow[b]{2}{*}{$\begin{array}{l}\text { Especies } \\
\end{array}$} & \multicolumn{4}{|c|}{1998} & \multicolumn{4}{|c|}{1999} & \multicolumn{4}{|c|}{2000} & \multicolumn{3}{|c|}{2001} & \multicolumn{2}{|c|}{2002} & \multirow[t]{2}{*}{ Total } \\
\hline & 1er & 2do & $3 e r$ & 4to & 1er & 2do & 3er & 4to & 1er & $2 \mathrm{do}$ & 3 er & 4to & $1 \mathrm{er}$ & 3 er & 4to & 2do & 3 er & \\
\hline Tenebrionidae (16) & 7 & 8 & 7 & 96 & 172 & 17 & 91 & 53 & 170 & 121 & 313 & 198 & 3788 & 312 & 441 & 91 & 428 & 6313 \\
\hline Carabidae (5) & 437 & 485 & 575 & 66 & 49 & 140 & 43 & 86 & 16 & 239 & 314 & 259 & 47 & 655 & 173 & 184 & 854 & 4622 \\
\hline Curculionidae (10) & 225 & 60 & 320 & 431 & 7 & 90 & 262 & 26 & 2 & 21 & 599 & 633 & 19 & 400 & 733 & 35 & 698 & 4561 \\
\hline Staphylinidae (7) & 6 & 9 & 108 & 351 & 31 & 75 & 136 & 37 & 5 & 54 & 99 & 161 & 19 & 195 & 245 & 59 & 546 & 2136 \\
\hline Elateridae (1) & 16 & 1 & 95 & 30 & 4 & 10 & 89 & 2 & o & 0 & 21 & 23 & 1 & 211 & 23 & 45 & 1349 & 1920 \\
\hline Coccinellidae (5) & 72 & 1 & 108 & 18 & 23 & 18 & 139 & 43 & 9 & 223 & 66 & 13 & o & 185 & 59 & 103 & 273 & 1353 \\
\hline Phalacridae (1) & 97 & 1 & 17 & 9 & 1 & 1 & 1 & 1 & o & 0 & 19 & 4 & o & 30 & 1 & 2 & 456 & 640 \\
\hline Mycetophagidae (2) & 21 & 24 & 196 & 24 & 46 & 24 & 70 & 13 & 1 & 6 & 12 & 6 & 3 & 18 & 1 & 22 & 4 & 491 \\
\hline Clambidae (1) & 0 & 0 & 4 & 1 & 117 & 14 & 36 & 41 & 28 & 6 & 9 & 13 & 3 & 3 & 5 & 1 & o & 281 \\
\hline Mordellidae (2) & 1 & o & 0 & 11 & 5 & 4 & 21 & 73 & 6 & o & o & 7 & 17 & 1 & 17 & 5 & o & 168 \\
\hline Chrysomelidae (1) & 31 & 10 & 32 & 33 & 4 & 1 & 2 & 2 & o & o & 4 & 3 & 0 & 18 & o & 1 & 4 & 145 \\
\hline Lathridiidae (2) & 4 & o & 2 & 3 & 2 & 12 & 24 & 13 & o & 12 & 2 & 16 & o & 3 & 4 & 6 & o & 103 \\
\hline Anthicidae (2) & o & o & 0 & 5 & 5 & 8 & 8 & 3 & 1 & 7 & 1 & 2 & 4 & 3 & 7 & 3 & 6 & 63 \\
\hline Bostrichidae (1) & o & o & o & o & 1 & 1 & 1 & 5 & o & 4 & o & o & o & 1 & o & 23 & 2 & 38 \\
\hline Pselaphidae (1) & 1 & 5 & o & 2 & o & o & o & o & o & 1 & o & o & o & 2 & o & 4 & 14 & 29 \\
\hline Nitidulidae (3) & 9 & o & 3 & o & o & 1 & o & o & o & 2 & 1 & o & o & o & o & 7 & o & 23 \\
\hline Trogidae (1) & o & o & o & o & o & o & o & o & o & o & o & 2 & 1 & 1 & o & 5 & 7 & 16 \\
\hline Histeridae (2) & 3 & o & 1 & 1 & 2 & o & 1 & o & 1 & o & o & o & 2 & o & o & o & o & 11 \\
\hline Colydiidae (1) & o & o & o & o & 1 & o & o & o & 1 & o & o & 1 & o & o & 2 & o & o & 5 \\
\hline Cleridae (1) & o & o & o & o & o & o & 1 & o & o & o & o & o & o & 0 & o & o & o & 1 \\
\hline Scarabaeidae (1) & o & o & o & o & o & o & o & 1 & o & o & o & o & o & o & o & o & o & 1 \\
\hline
\end{tabular}

